\begin{tabular}{|c|c|c|c|c|c|c|c|c|c|c|c|c|c|c|}
\hline $\begin{array}{l}\text { Arg. } \\
0.023\end{array}$ & $\begin{array}{c}\text { Burrau } \\
0.02300\end{array}$ & $\begin{array}{l}\text { richtig } \\
2300\end{array}$ & $\begin{array}{l}\text { Arg. } \\
0.204\end{array}$ & $\begin{array}{c}\text { Burran } \\
0.20541 .\end{array}$ & $\begin{array}{l}\text { richtig } \\
0542\end{array}$ & $\begin{array}{c}\text { Arg. } \\
0.444\end{array}$ & $\begin{array}{c}\text { Burrau } \\
0.45^{873}\end{array}$ & $\begin{array}{l}\text { richtig } \\
5873^{\circ}\end{array}$ & $\begin{array}{l}\text { Arg. } \\
0.639\end{array}$ & $\begin{array}{c}\text { Burrau } \\
0.6833^{8}\end{array}$ & $\begin{array}{l}\text { richtig } \\
833^{8} .\end{array}$ & $\begin{array}{l}\text { Arg. } \\
0.854\end{array}$ & $\begin{array}{c}\text { Burrau } \\
0.96165\end{array}$ & $\begin{array}{l}\text { richtig } \\
6 \times 66\end{array}$ \\
\hline 0.024 & $2400^{\circ}$ & 2400 & 0.212 & I $359^{\circ}$ & 1359 & 0.445 & 5983 & 5933. & 0.644 & 8944 & $8944^{\circ}$ & 0.862 & 7278 & 7279 \\
\hline .042 & 4201 & 4201 & 0.253 & $5570^{\circ}$ & $557 \mathrm{I}$ & 0.453 & 6865 & $6865^{\circ}$ & 0.646 & 9187. & 9 I 88 & 0.872 & 867.9 & \\
\hline .047 & 4702 & $470 \mathrm{I}^{\circ}$ & 0.262 & & $650 x$ & 0.454 & $6975^{\circ}$ & $69 \% 6$ & 0.666 & $0.7 \pm 633$ & 1634 & 0.897 & 1.02222 & 222 \\
\hline .068 & $6805^{\circ}$ & 6805 & 0.265 & 68 I I & $68 \mathrm{Ir}$ & 0.459 & & $75 \% 9$ & $0.67 \mathrm{I}$ & $2249^{\circ}$ & 2250 & 0.904 & 3226 & 3225 \\
\hline 0.074 & 7406 & $74 \circ 7$ & 0.283 & 8679 & $8679^{\circ}$ & 0.532 & 0.55745 & 5745 & 0.675 & $2743^{\circ}$ & 2744 & 0.912 & 4378 & 4379 \\
\hline 0.098 & 9816 & 98 I 5 & 0.311 & 0.31603 & 1604 & 0.566 & 9670 & 9671 & 0.689 & 4482 & 4482 & 0.919 & $5393^{\circ}$ & 5393 \\
\hline 0.104 & $0.104 \mathrm{I} 8 \cdot$ & 04 I 9 & 0.319 & $2443^{\circ}$ & 2444 & 0.567 & 9787 & 9787. & 0.749 & 0.82102 & 2102 & 0.923 & $5975^{\circ}$ & 5975 \\
\hline 0.124 & $243 \mathrm{I}$ & 2432 & 0.361 & $6889^{\circ}$ & 6889 & 0.594 & $0.62955^{\circ}$ & 2955 & $0.75^{2}$ & $2490^{\circ}$ & 2491 & 0.939 & $8320^{\circ}$ & 8320 \\
\hline o. 133 & 38 & 3339 & 0.368 & 7636. & $76_{3} 6$ & 0.607 & 4496 & 4497 & 0.762 & $379 \mathrm{I}$ & $379 \mathrm{I}^{\circ}$ & 0.943 & 89 .0. & $891 x$ \\
\hline 0.179 & & & $0.3^{8} 5$ & $945^{8}$ & $945^{8}$ & 0.608 & 4615. & 4616 & 0.763 & $392 \mathrm{I}^{\circ}$ & 3922 & 0.953 & I. $10394^{\circ}$ & 0395 \\
\hline 0.187 & $8809^{\circ}$ & 8809 & 0.403 & 0.41400 & I $399^{\circ}$ & $0.6 \times 4$ & $3 I$ & & 0.803 & $92 \times 2$. & 9212 & 0.954 & $0543^{\circ}$ & 0544 \\
\hline 0.196 & 9726 & $9725^{\circ}$ & 0.419 & $313^{6}$ & $3 \times 37$ & 0.615 & $545^{\circ}$ & $545 \mathrm{I}$ & & 0.92591 & $259^{\circ}$ & 0.959 & I 290 & I $290^{\circ}$ \\
\hline 0.197 & 9828 & 9827. & 0.443 & $57^{6} 3$ & 5763. & 0.633 & 76 I 2. & 7613 & 0.853 & 5927 & 6027 & 0.965 & $2190^{\circ}$ & 2190 \\
\hline
\end{tabular}

Nur bei sin $0.853 i$ ist der Fehler bedeutend, nämlich eine Einheit der dritten Stelle. Bei sin $0.644 i$ und $\sin 0.959 i$ beträgt der wirkliche Fehler eine halbe Einheit der fünften Stelle; bei allen übrigen übersteigt er nur wenig $1 / 4$ Einheit dieser Stelle.

Wien, Ig I 5 Februas.

M. Esch, S. J.

\title{
Mitteilungen über Kometen.
}

Komet I9I5 a (Mellish). Herr Prof. Strömgren teilt folgende von Herrn Observator 7. Braae erhaltene Beobachtungen des Kometen am Refraktor der Sternwarte zu Kopenhagen mit:

\begin{tabular}{|c|c|c|c|c|}
\hline 1915 & M. Z. Kopenh. & $\alpha$ app. & ठ app. & Vergl. \\
\hline Nov, I I & $13^{h} 46^{m} 45^{s}$ & $4^{h} 55^{\mathrm{m}} \quad 9^{\mathrm{s}} 83$ & $-6^{\circ} 21^{\prime} 33^{\prime \prime} \cdot 2$ & I $2, I 2$ \\
\hline I 5 & 14230 & $\begin{array}{lll}4 & 45 & \text { I } 3.08\end{array}$ & $-4 \quad 5427.9$ & 6,6 \\
\hline
\end{tabular}
hiernach: $-3^{\mathrm{s}} \mathrm{o}^{\prime}$.

Komet r9i5d (Mellish). Das Lick Observ. Bull. 273 enthält eine neue von Dr. S. Einarsson und $D$. Alter aus Beobachtungen vom 20., 2 I. und 23. Sept. berechnete Bahn des Kometen. Die Elemente sind:

$$
\begin{aligned}
& T={ }_{1915} \text { Okt. I } 3.3959 \text { m. Z. Gr. } \\
& \omega=1 \text { I } 8^{\circ} 50^{\prime} 3^{\prime \prime} \\
& \delta 8=774252 \\
& i=533241 \\
& \log q=9.64669 \\
& \text {. }
\end{aligned}
$$

$$
\begin{aligned}
& \text { Ephemeride für } \text { I }^{\text {h }} \mathrm{m} \text {. Z. Gr. } \\
& \text { I9I5 } \alpha \text { vera overa } \log \Delta \quad H
\end{aligned}
$$

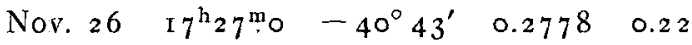

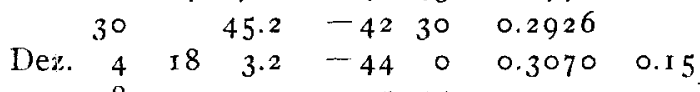

$$
\begin{aligned}
& \begin{array}{lllll}
8 & 21.0 & -45 & 15 & 0.321 \mathrm{I}
\end{array}
\end{aligned}
$$

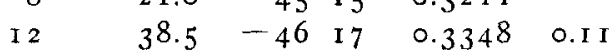

$$
\begin{aligned}
& \begin{array}{lllll}
16 & 55.7 & -47 & 8 & 0.3480
\end{array} \\
& \begin{array}{lllllll}
20 & \text { I } 9 & \text { I } 2.5 & -47 & 49 & 0.3608 & 0.08
\end{array} \\
& \begin{array}{llllll}
24 & 28.9 & -48 & 20 & 0.3731
\end{array} \\
& \begin{array}{lllllll}
28 & \text { I9 } & 44.9 & -48 & 44 & 0.3849 & 0.06
\end{array}
\end{aligned}
$$

Helligkeit für Sept. 2 I $\mathrm{H}=1.00$.

Bei der Redaktion sind keine direkte Nachrichten über den Kometen eingegangen, und es war deshalb eine Prüfung und Verbesserung der in $\mathrm{Nr} .48 \mathrm{I} 7$ mitgeteilten Bahn nicht ausführbar. Der ihr zugrunde liegende erste Ort war, wie sich jetzt ergibt, nur ein genähert richtiger.

Kiel, I9I 5 Nov. 24.

H. Kobold.

\section{Zodiakallicht.}

Am 17. Nov. I915 wurde in Neuschloß, $\lambda={ }_{1^{\mathrm{h}}} \mathrm{I}^{\mathrm{m}} 5 \mathrm{I}^{\mathrm{s}}$ östl. v. Greenw., $\varphi=+46^{\circ} 55^{\prime} 9^{\prime \prime}$, von $16^{\mathrm{h}}$ bis $17^{\mathrm{h}} 15^{\mathrm{m}}$ ein Zodiakallicht beobachtet, das ungefähr um $16^{\mathrm{h}} 50^{\mathrm{m}}$ das Maximum der Helligkeit and Ausdehnung erreichte und zu der Zeit mindestens viermal so hell war wie die Milchstraße im Stier.

Neuschloß, I9 I 5 Nov. I g.
Es wurden folgende Koordinaten gefunden:

Breite der Basis: $\alpha=187^{\circ}$ bis $\alpha=200^{\circ}$.

Ferner $\alpha=175^{\circ} \delta=+4^{\circ}$

$$
\alpha=\mathrm{I} 84^{\circ} \quad \delta=+\mathrm{I}^{\circ}
$$

welche Punkte einem Schnitt etwa in der halben Höhe des Lichtkegels entsprechen. Spitze: $\alpha={ }_{15} 6^{\circ} \delta=+{ }_{1} 6^{\circ}$.

\section{Fosef Sedläcek.}

5 Astraea. Korrektion der Oppositionsephemeride des B. f. I9 15 Nov. 1o + 3'7 + I 8'. Luther.

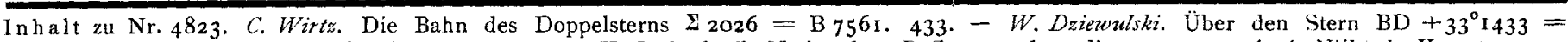
26.19I2 Geminorum. 435. - H. Lualendorff. Notiz iber R Coronae borealis. 439. - A. A. Nijland. Komet I9I5 a (Mellish). 441. - F. Holetschek. Beobachtungen uber die Größe und Helligkeit des Kometen I9I5 a (Mellish). 443. C. Hoffmeister. Helligkeitsschätzungen am Kometen I9I3f (Delavan). 443. - P. Guthnick. R. Prager. Anzeige neuer Veränderlicher. 443, - R. Castro. Observaciones del cometa I9I3f (Delavan). 445. - M. Esch. Berichtigung zu Burrau, Tafeln der Funktionen Cosinus und Sinus. 445. - H. Kobold. Mitteilungen über Kometen. 447. - F. Sedlácek. Zodiakal. licht. 447. - W. Luther. 5 Astraea. 447. 